\title{
The revolving door of mental, neurological, and substance use disorders re-hospitalization in rural KwaZulu-Natal Province, South Africa.
}

\author{
Andrew Tomita ${ }^{1}$, Yoshan Moodley²
}

1. Africa Centre for Health and Population Studies, University of KwaZulu-Natal, Durban, South Africa.

2. Discipline of Anesthesiology and Critical Care Medicine, Nelson R. Mandela School of Medicine, University of KwaZulu-Natal, South Africa.

\begin{abstract}
Objective: Little is known about the extent of mental, neurological and substance-use (MNS) disorders re-hospitalization in South Africa. We examined the extent of one-year MNS re-hospitalization (MNS-R) in a rural South African primary health care facility (PHCF).

Methods: We conducted a retrospective analysis of hospital administrative data from 10,525 adults discharged from a rural PHCF in KwaZulu-Natal Province, South Africa. Chi-squared tests were utilized to describe MNS-R within one year of an index hospital admission in individuals with MNS, with a sub-analysis also being conducted to describe schizophrenia re-hospitalization (S-R).

Results: The prevalence of MNS and schizophrenia recorded at an index hospitalization was 5\% and 1\%, respectively. A total of 44/67 (66\%) individuals with a diagnosis of MNS at the index hospitalization were classified as having MNS-R during oneyear follow-up period. Half of those diagnosed with schizophrenia at the index hospitalization (6/12 patients) were classified as having S-R during one-year follow-up period. There was a significant association between re-hospitalization outcomes (MNS-R and $\mathrm{S}-\mathrm{R})$ and MNS $(\mathrm{p}<0.01)$ or schizophrenia diagnosis $(\mathrm{p}<0.01)$ at index baseline hospitalization.

Conclusion: The extent of MNS-R and S-R remains relatively high in rural South Africa, and needs further health systems strengthening to prevent revolving door occurrences.
\end{abstract}

Keywords: Rural mental health, severe mental illness, administrative data, South Africa.

DOI: http://dx.doi.org/10.4314/ahs.v16i3.23

Cite as: Tomita A, Moodley Y. The revolving door of mental, neurological, and substance use disorders re-hospitalization in rural KwaZulu-Natal Province, South Africa. Afri Health Sci 2016;16(3): 817-821. DOI: http://dx.doi.org/10.4314/abs.v16i3.23

\section{Introduction}

Despite evidence that mental, neurological and substance-use (MNS) disorders accounts for a substantial proportion of premature mortality and disability globally ${ }^{1}$, a deficit of services to treat the condition is a persistent challenge throughout sub-Saharan Africa ${ }^{2}$, including South Africa. While funding for mental health services is acknowledged to receive a disproportionately small proportion of health budgets across many developing coun-

\section{Corresponding author: \\ Andrew Tomita, \\ Africa Centre for Health and Population Studies, University of KwaZulu-Natal, Durban, South Africa. P.O.Box 198, Mtubatuba, 3935, South Africa. \\ Tel: +27 (0)31-260-4321 (South Africa) \\ Fax: +27 (0)31-260-4322 (South Africa). \\ E-Mail: tomita@ukzn.ac.za}

tries $^{3}$, policy-makers and researchers seldom focus on this regional and urban-rural resource inequity in an effort to address such conditions. In South Africa, post-apartheid urban-rural inequality in health services remains an ongoing challenge $\mathrm{e}^{4}$, and nowhere is this more evident and challenging than for mental health services in KwaZulu-Natal (KZN) Province ${ }^{5}$.

For certain MNS disorders, such as schizophrenia ${ }^{6}$ and psychosis $^{7}$, relapse is a disturbing and frequent occurrence, often due to treatment cessation. With each subsequent relapse, refractoriness may arise ${ }^{8}$, and even when pharmacologic treatment is restored, individuals with schizophrenia may not be able to return to the previous level of social functioning 9 . Approximately two thirds of people diagnosed with schizophrenia relapse ${ }^{10}$, with unmet needs for mental health treatment in rural areas being well documented in a large U.S.-based National Comorbidity Survey Replication study ${ }^{11}$. Cohort studies of 
non-tertiary rural patients with MNS, including schizophrenia, remains limited. According to a South African study, approximately half of the inpatients interviewed at a KZN tertiary psychiatric hospital reported experiencing mental health related hospitalilization one year prior to initial [index] admission ${ }^{12}$. Our study investigated the extent of MNS re-hospitalization (MNS-R) and schizophrenia re-hospitalization (S-R) among a cohort of rural patients accessing mental health care services at a rural primary healthcare facility.

\section{Methods}

\section{Study design, setting, population, and data source}

This study utilized the longitudinal data from the Hospital Information System (HIS) for a government hospital providing services at primary care level located approximately $250 \mathrm{~km}$ North of the largest city in KZN. The hospital oversees 17 fixed clinics, and services a large and predominantly rural sub-district population of over 200,000 mainly Zulu speaking individuals within an area of $1400 \mathrm{~km}^{2}$. The general population of the district as well as details about the Health Information System have been described in detail elsewhere $e^{13,14}$.

The dataset contains demographic information and International Classification of Disease version 10 (ICD-10) codes assigned by healthcare professionals with a clinical and coding background. Information on 24,262 inpatient individuals of all ages between January 2011 and February 19, 2015 were available. Record selection criteria were adults 15 years and above discharged from the hospital prior to February 19, 2014 (to allow minimum one-year observation), with surgical adult patients being excluded from the study. The dataset is de-identified and publicly available, and the study was approved by the Biomedical Research Ethics Committee of the University of KwaZulu-Natal, South Africa.

\section{Measures}

The primary outcome was MNS-R [discharge diagnosis ICD-10 codes: F00-F99, G30 and G40], where re-hospitalization was defined as a subsequent hospital admission for the same patient that occurred between 24 hours and one year following discharge from the index hospitalization. A study indicated that reports of hospitalization obtained from administrative databases are a particularly useful proxy for relapses in population settings ${ }^{15}$. The main study predictors were MNS [discharge diagnosis ICD-10 codes: F00-F99, G30 and G40] diagnosis during index hospitalization. In addition to F00-F09 (mental and behavioral disorders), our study included Alzheimer's (ICD-10 G30) and epilepsy (G40) to operationalize MNS, consistent with a previous publication ${ }^{16}$. As described previously, the relapse among individuals with debilitating disorders, such as schizophrenia, is pronounced. A sub-analysis to describe the risk of schizophrenia rehospitalization (S-R) within one year of an index hospital admission in individuals with schizophrenia [discharge diagnosis ICD-10 codes: F20] was also conducted.

\section{Statistical analysis}

First, demographic characteristics and mental health diagnoses at the index hospitalization were established using descriptive statistics. Second, the proportion of individual with MNS/schizophrenia re-hospitalized with the same diagnoses category during a one-year followup period was computed. The association between index hospitalization and re-hospitalization diagnoses in MNS/ schizophrenia was explored using $\chi^{2}$. A p-value $<0.05$ was considered statistically significant. STATA 14 was utilized to check for sample duplication (and analyses).

\section{Results}

The demographic characteristics and mental health diagnoses at the index hospitalization are described in Table 1. 


\section{Table 1. Demographic characteristics and mental health diagnoses at index (baseline) hospitalization}

\begin{tabular}{|c|c|c|c|c|}
\hline \multirow[t]{2}{*}{ Variable } & \multicolumn{2}{|c|}{ All adults } & \multicolumn{2}{|c|}{ Study cohort } \\
\hline & $\mathrm{n}$ & $\%$ & $\mathrm{n}$ & $\%$ \\
\hline \multicolumn{5}{|l|}{ Gender: } \\
\hline Male & 3,774 & 35.9 & 462 & 41.6 \\
\hline Female & 6,751 & 64.1 & 650 & 58.5 \\
\hline \multicolumn{5}{|c|}{ Age categories: } \\
\hline $15-24$ & 2,898 & 27.5 & 186 & 16.7 \\
\hline $25-34$ & 3,055 & 29.0 & 302 & 27.2 \\
\hline $35-44$ & 1,711 & 16.3 & 212 & 19.1 \\
\hline $45+$ & 2,861 & 27.2 & 412 & 37.1 \\
\hline \multicolumn{5}{|c|}{ MNS diagnosis (based on ICD-10 codes F00-F99, G30 and G40): } \\
\hline No & 10,045 & 95.4 & 1031 & 92.7 \\
\hline Yes & 480 & 4.6 & 81 & 7.3 \\
\hline \multicolumn{5}{|c|}{ Schizophrenia (based on ICD-10 codes F20): } \\
\hline No & 10,440 & 99.2 & 1102 & 99.1 \\
\hline Yes & 85 & 0.8 & 10 & 0.9 \\
\hline
\end{tabular}

Of the 10,525 adults in the dataset, 6,751 (64\%) were female, with a mean $( \pm S D)$ age of $37( \pm 18)$ years. The prevalence of MNS and schizophrenia in the baseline sample were $480(5 \%)$ and $85(1 \%)$ respectively. When the dataset was limited to a study cohort who experience re-hospitalization from all causes within a year from index hospitalization, the sample size was 1,112 adults. Of these adults in the baseline analyses, 650 (59\%) were female, with a mean $( \pm S D)$ age of $42( \pm 18)$ years. The prevalence of MNS and schizophrenia in the limited baseline sample were $81(7 \%)$ and $10(1 \%)$ respectively.
A total of $44 / 67(66 \%)$ individuals with a diagnosis of MNS at the index hospitalization were re-hospitalized for the same condition during the one-year follow-up period. Similarly, a total of $6 / 12(50 \%)$ individuals with a diagnosis of schizophrenia at the index hospitalization were re-hospitalized for the condition during the one-year follow-up period. In both re-hospitalization outcomes (i.e. MNS-R and S-R), there was significant association with MNS and schizophrenia diagnosis at index baseline hospitalization $(\mathrm{p}<0.01)$ based on $\chi^{2}$ testing (Table 2$)$

\section{Table 2. Association between index hospitalization and re-hospitalization diagnoses}

\begin{tabular}{|c|c|c|c|c|c|c|c|c|}
\hline \multicolumn{2}{|c|}{ Index hospitalization } & \multicolumn{2}{|c|}{$\begin{array}{l}\text { Re-hospitalization but not } \\
\text { due to MNS (A) } \\
\text { due to schizophrenia (B) }\end{array}$} & \multicolumn{2}{|c|}{$\begin{array}{c}\text { Re-hospitalization } \\
\text { due to MNS (A) } \\
\text { due to schizophrenia (B) }\end{array}$} & \multirow[t]{2}{*}{ df, $\chi^{2}$} & \multirow[t]{2}{*}{$\mathrm{p}$} & \multirow{2}{*}{$\frac{\text { Total }}{n}$} \\
\hline \multirow{3}{*}{ (A) } & & $\mathrm{n}$ & $\%$ & $\mathrm{n}$ & $\%$ & & & \\
\hline & No MNS & 1,008 & 96.5 & 37 & 3.5 & 1,359 & $<0.001$ & 1045 \\
\hline & MNS & 23 & 34 & 44 & 66 & & & 67 \\
\hline \multirow[t]{2}{*}{ (B) } & $\begin{array}{c}\text { No } \\
\text { schizophrenia }\end{array}$ & 1,096 & 99.6 & 4 & 0.4 & 1,328 & $<0.001$ & 1,100 \\
\hline & Schizophrenia & 6 & 50 & 6 & 50 & & & 12 \\
\hline
\end{tabular}




\section{Discussion}

Two thirds of patients with MNS at their index hospital admission were re-hospitalized within the one year follow-up period in this study. This suggests a 'revolving door' phenomenon among this vulnerable population, which warrants the attention of hospital clinicians and community-based services. Bearing in mind that there are few studies describing re-hospitalization in rural populations, recent studies of severe mental illness from developing and developed nations reported one-year risk of re-hospitalization to range from $40-52 \%{ }^{17-19}$, within which our estimate falls.

The major caveat or limitation is the extent of potential under-reporting/diagnosis of MNS and schizophrenia in our study dataset, with the number of cases (who experienced re-hospitalization within a year from index hospitalization) being particularly limited, which an examination of re-hospitalization outcomes using narrow categories of disorders within MNS. It may be speculated that the extent of MND found in our study should be have been considerably higher, as it is based on clinical samples, and that the scarcity of mental health services in rural areas makes hospitalization in other facilities unlikely. The possible explanation with respect to our underestimates is a general shortcoming associated with the use of administrative data ${ }^{20}$, as well as limitations in validity, coding verification, and misclassification of diagnoses.

Efforts to prevent subsequent revolving door phenomenon of mental health re-hospitalization will require more than the customary hospital discharge plan. For individuals with MNS and schizophrenia, specifically for the first time, the period after hospital discharge can be a difficult transition time, given the lack of and fragmented nature of mental health services in the community. This period may require care coordination interventions that promote access to formal services, hospital discharge plans to be implemented, and systematic links to broader social system fostered that allow person with MNS to receive ongoing support in the community and avoid unnecessary re-hospitalization. There are several promising randomized controlled trials underway in LMIC (low and middleincome countries) settings. These include Task Sharing for the Care of Severe Mental Disorders in a low-income country (TaSCS) in Ethiopia ${ }^{21}$, and time-limited Criti-

African Health Sciences Vol 16 Issue 3, September 2016 cal Time Intervention-Task Shifting (CTI-TS) in Brazil, Chile, and Argentina ${ }^{22}$. Greater effort need to be places on attempts to advance the lives of individuals with MNS and severe mental disorders by promoting sustainable community-based mental health care. Despite the limitations of our study, as a first step, our findings uniquely identify the challenges related to revolving door phenomenon among individuals with MNS in a rural South African population, highlighting the need for much work to be done in rural mental health services.

\section{Acknowledgement:}

Andrew Tomita was supported by NIH Research Training Grant (R25TW009337), funded by the Fogarty International Center and the National Institute of Mental Health as well as South African Medical Research Council (MRC) flagship grant (MRC-RFA-UFSP-01-2013/ UKZN HIVEPI). Yoshan Moodley was supported by a postdoctoral fellowship awarded by the South African National Research Foundation (NRF). The content of this manuscript is solely the responsibility of the authors and does not necessarily represent the official views of the NIH, NRF or the MRC. We acknowledge Luvaseni Pillay who assisted in this article.

\section{Conflict of interest}

None to declare.

\section{References}

1. Vos T, Flaxman AD, Naghavi M, Lozano R, Michaud C, Ezzati M, et al. Years lived with disability (YLDs) for 1160 sequelae of 289 diseases and injuries 1990-2010: a systematic analysis for the Global Burden of Disease Study 2010. Lancet. 2012; 380:2163-96.

2. Collins PY, Musisi S, Frehywot S, Patel V. The core competencies for mental, neurological, and substance use disorder care in sub-Saharan Africa. Glob Health Action. 2015; 8:26682.

3. Saxena S, Thornicroft G, Knapp M, Whiteford H. Resources for mental health: scarcity, inequity, and inefficiency. Lancet. 2007; 370:878-889.

4. Hassim A, Heywood M, Berger J. Health \& democracy: A guide to human rights, health law and policy in postapartheid South Africa. Cape Town: SiberInk, 2007.

5. Burns JK. Mental health services funding and development in KwaZulu-Natal: a tale of inequity and neglect. S Afr Med J. 2010; 100:662-6. 
6. Robinson D, Woerner MG, Alvir JM, Bilder R, Goldman R, Geisler S, et al. Predictors of relapse following response from a first episode of schizophrenia or schizoaffective disorder. Arch Gen Psychiatry. 1999; 56:241-7.

7. Alvarez-Jimenez M, Priede A, Hetrick SE, Bendall S, Killackey E, Parker AG, et al. Risk factors for relapse following treatment for first episode psychosis: a systematic review and meta-analysis of longitudinal studies. Schizophr Res. 2012; 139:116-128.

8. Lieberman JA, Alvir JM, Koreen A, Geisler S, Chakos M, Sheitman B, et al. Psychobiologic correlates of treatment response in schizophrenia. Neuropsychopharmacology. 1996; 14:13S-21S.

9. Wyatt RJ. Research in schizophrenia and the discontinuation of antipsychotic medications. Schizophr Bull. 1997; 23:3-9.

10. Smith T, Thomas S, Christi W. Schizophrenia (Maintenance Treatment). Am Fam Physician. 2010; 82:338.

11. Wang PS, Lane M, Olfson M, Pincus HA, Wells KB, Kessler RC. 2005. Twelve-month use of mental health services in the United States: results from the National Comorbidity Survey Replication. Arch Gen Psychiatry. 2005; 62:629-40.

12. Tomita A, Burns JK, King H, Baumgartner JN, Davis GP, Mtshemla S, et al. Duration of untreated psychosis and the pathway to care in KwaZulu-Natal, South Africa. J Nerv Ment Dis. 2015; 203:222-5.

13. Garrib A, Stoops N, McKenzie A, Dlamini L, Govender T, Rohde J, et al. An evaluation of the District Health Information System in rural South Africa. $S$ Afr Med J. 2008; 98:549-52.

14. Tanser F, Hosegood V, Barnighausen T, Herbst K, Nyirenda M, Muhwava W, et al. Cohort profile: Africa Centre Demographic Information System (ACDIS) and population-based HIV survey. Int J Epidemiol. 2008; 37:956-962.

15. Addington DE, Patten SB, McKenzie E, Addington J. Relationship between relapse and hospitalization in firstepisode psychosis. Psychiatr Serv. 2013; 64:796-9.

16. World Health Organization. Fact Sheet: Mental disorders. 2014. Accessed from http://www.who.int/mediacentre/factsheets/fs396/en/

17. Zhang J, Harvey C, Andrew C. Factors associated with length of stay and the risk of readmission in an acute psychiatric inpatient facility: a retrospective study. Aust N ZJ Psychiatry. 2011; 45:578-85.

18. Loch AA. Stigma and higher rates of psychiatric rehospitalization: Sao Paulo public mental health system. Rev Bras Psiquiatr. 2012; 34:185-92.

19. Schennach R, Obermeier M, Meyer S, Jager M, Schmauss M, Laux G, et al.. Predictors of relapse in the year after hospital discharge among patients with schizophrenia. Psychiatr Serv. 2012; 63:87-90.

20. Grosse SD, Boulet SL, Amendah DD, Oyeku SO. Administrative data sets and health services research on hemoglobinopathies: a review of the literature. Am J Prev Med. 2010. 38:S557-67.

21. University of Cape Town. Task sharing for the care of severe mental disorders in a low-income Country (TaSCS). In ClinicalTrials.gov [Internet], Bethesda, MD: National Library of Medicine (US). 2015. Retrieved from http://clinicaltrials.gov/show/ NCT02308956. NLM Identifier: NCT02308956

22. da Silva TFC, Lovisi G, Tavares Cavalcanti M, Dahl C, Conover S, Valencia E, et al. Critical time intervention-Task shifting: A new psychosocial intervention for people with severe mental illness in Latin America. Revista Psiquiatria Clínica. 2013; 40:243. 